Original Article

\title{
Age-related differences in control of a visuomotor coordination task: a preliminary study
}

Young Uk Ryu, PT, PhD ${ }^{1)}$, Kyu-Ho Lee, MS, PT ${ }^{1)}$, Hocheol Lee, PhD²), Jungsik Park, PhD ${ }^{3)^{*}}$

1) Department of Physical Therapy, College of Medical Science, Catholic University of Daegu, Republic of Korea

2) School of Mechanical and Automotive Engineering, Catholic University of Daegu, Republic of Korea

3) Center for Medical Humanities and Convergent Contents, Ajou University: Worldcup-ro 206, Yeongtong-gu, Suwon, Republic of Korea

\begin{abstract}
Purpose] The purpose of the current study was to examine age-related differences in control of a perception-action coordination skill. We adapted a visuomotor tracking experiment requiring various coordination patterns between a limb's motion and an external signal. [Subjects and Methods] A total of 12 subjects (6 elderly and 6 young) voluntarily participated in the study. The experimental session consisted of 3 trials for 3 different relative phase patterns: $0^{\circ}, 90^{\circ}$, and $180^{\circ}$, defined by the relationship between the online visual feedback of the joystick motion and the white dot signal. [Results] The $0^{\circ}$ and $180^{\circ}$ tracking patterns were stable compared with the $90^{\circ}$ tracking pattern for both age groups. The present results also showed that the elderly subjects were less stable than were young subjects for all tracking patterns. [Conclusion] The intrinsic coordination dynamics predicted by the Haken-Kelso-Bunz (HKB) mathematical model did not change with age, whereas utilization of visual feedback information declined overall. Further research is needed regarding methods for increasing utilization of visual feedback information from the perspective of rehabilitation.

Key words: Perception-action, Coordination, Aging
\end{abstract}

(This article was submitted Nov. 30, 2015, and was accepted Jan. 8, 2016)

\section{INTRODUCTION}

Visuomotor tracking tasks have been used to investigate the underlying mechanisms of perception-action that support the coordination of a movement to an environmental event when tracking a moving object based on visual information ${ }^{1,2)}$.

In visuomotor tracking tasks examining the nature of such perception-action coordination skills, actors track a rhythmic external signal in various phase relationships between the external signal and a limb $b^{3,4)}$.

Previous investigations utilizing the visuomotor tracking paradigm demonstrated that an in-phase tracking pattern (external signal and limb motion moving together in the same direction) is more stable than an anti-phase pattern (external signal and limb motion moving in opposite directions), and that relative phase patterns between the two relative phases are difficult to track ${ }^{3,4)}$. Based on such differential stability among relative phase patterns, Haken et al. developed a mathematical model (called the HKB model) and named it 'intrinsic coordination dynamics'5).

Many previous investigations demonstrated that increased age leads to a decrease in perceptual motor skill on various experimental tasks ${ }^{6-8)}$. Such studies adapting perceptual motor tasks demonstrated that elderly people tend to rely more on external signals than do young people ${ }^{9,10}$. Moreover, elderly people use more time in processing visual information than do young people, resulting in a marked decline in motor performance ${ }^{11,12)}$. However, no such studies have investigated the

*Corresponding author. Jungsik Park (E-mail: jspark@ajou.ac.kr)

(C2016 The Society of Physical Therapy Science. Published by IPEC Inc.

This is an open-access article distributed under the terms of the Creative Commons Attribution Non-Commercial No Derivatives (by-nc-nd) License $<$ http://creativecommons.org/licenses/by-nc-nd/4.0/>. 
effects of aging on the nature of perception-action coordination in a perceptual motor task.

The perceptual motor system improves or maintains qualities of tracking performance by correcting unstable movements ${ }^{4}$. Corrective movements in such visuomotor tracking tasks are characterized by directional changes in the velocity profile of the tracking trajectory and are measured by an increase in power (referred to as intermittency) in a frequency band higher than the target signal's frequency ${ }^{13}$. A recent study of Ryu and Buchanan ${ }^{4}$ showed that the intermittency measurement may be an important tool for understanding a perception-action coordination skill.

The purpose of the current study was to examine age-related differences in control of a perception-action coordination skill. We adapted a visuomotor tracking experiment that required various coordination patterns between a limb's motion and an external signal. Based on a previous finding ${ }^{14,15)}$, it is expected that tracking performance in various coordination patterns would be degraded for elderly people compared with young people. An important question for the present study was to identify the effect of aging on the intrinsic coordination dynamics. If the intrinsic coordination dynamics are an inherent part of human motor coordination, the differential stability among the coordination patterns would be expected to remain for elderly people.

\section{SUBJECTS AND METHODS}

The protocol and consent form for this study were approved by the Institutional Review Board of Catholic University of Daegu. All subjects signed a written consent prior to participation. A total of 12 subjects voluntarily participated in the study. Age group demographics are presented in Table 1. Elderly subjects were required to achieve a minimum Mini-Mental State Exam score of 25 to participate and were provided with a health history questionnaire to exclude those who may have had a condition affecting motor performance. All subjects were self-reported to be right-handed with normal or corrected-tonormal vision.

Subjects sat on a height-adjustable chair with the right forearm comfortably placed on a custom-made forearm rest with a cuff to secure the forearm, allowing comfortable use of a force-feedback joystick (Logitech ${ }^{\circledR}$ Force 3D Pro). Subjects grasped the joystick, which sat on a table. The force feedback function of the joystick was disabled to minimize the resistance of the joystick. The joystick was placed in a box covering the subjects' hand and forearm, so that subjects could not see their joystick motions. A computer monitor was placed at a distance of approximately 0.6 meters from the subject's face and was used to display two dots. The two dots were displayed with two different colors, red and white, within a single window display ( $15 \mathrm{~cm}$ long $\times 5 \mathrm{~cm}$ high) with a black background. Each dot was approximately $4 \mathrm{~mm}$ in diameter, and was displayed one above the other (10 mm gap). The top red dot was under the control of the computer, and traversed a path approximately $125 \mathrm{~mm}$ across from left to right. The oscillation frequency for the red dot was set at $0.333 \mathrm{~Hz}$ with a total of 8 cycles per trial. The bottom white dot was controlled by the subject, using the joystick. Subjects were asked to move the joystick from side to side in order to move the white dot, representing the subjects' motion.

Prior to the main experiment, subjects were given 4 familiarization trials with the production of the joystick motion displayed on the computer monitor. Subjects were asked to track the white dot signal in both a $0^{\circ}$ relative phase (two dots moving in the same direction) and a $180^{\circ}$ relative phase (two dots moving in the opposite direction) tracking pattern. For these trials ( 2 trials each), the oscillation frequency was set at $0.25 \mathrm{~Hz}$ with a total of 8 cycles in a trial. This oscillation frequency was slower than the one used in the experimental sessions $(0.333 \mathrm{~Hz})$, to reduce the practice effect on tracking before the experiment. The experiment was conducted immediately after the familiarization trials.

The experimental session consisted of 3 trials for 3 different relative phase patterns: $0^{\circ}, 90^{\circ}$, and $180^{\circ}$, defined by the relationship between the online visual feedback of the joystick motion and the white dot signal. The $90^{\circ}$ relative phase pattern was in-between the $0^{\circ}$ and $180^{\circ}$ patterns. Before performing each relative phase pattern, the required relative phase pattern was shown to a subject. The computer-generated signal was represented with the white dot, while the signal that the subjects were supposed to reproduce with joystick motion was represented with the red dot. The assigned subject's joystick signal lagged behind the computer generated white dot signal in the $90^{\circ}$ relative phase pattern. Subjects were instructed to watch the pattern represented by the two dots and to try to reproduce the pattern. After watching one of the relative phase patterns, subjects immediately performed 3 consecutive trials for the assigned relative phase pattern. The three relative phase patterns were performed in a random order.

Prior to data analysis, a dual-pass Butterworth Filter was applied to the joystick trajectories, with a cutoff frequency of $10 \mathrm{~Hz}$. The first cycle of motion was dropped and considered as an adaptation phase, so that 7 cycles were used to analyze each trial. A continuous relative phase was computed to characterize the temporal tracking relationship between the computergenerated signal and the joystick motion. For both the computer-generated signal $\left(\Theta_{\text {computer }}\right)$ and joystick motion $\left(\Theta_{\text {joystick }}\right)$,

Table 1. General characteristics of the age groups

\begin{tabular}{lccc}
\hline Group & Age $($ mean $\pm \mathrm{SD})$ & Age range $(\mathrm{yrs})$ & MMSE $($ mean $\pm \mathrm{SD})$ \\
\hline Young $(\mathrm{N}=6)$ & $22.8 \pm 2.14$ & $20-26$ & $\mathrm{n} / \mathrm{a}$ \\
Old $(\mathrm{N}=6)$ & $66.8 \pm 0.98$ & $65-68$ & $28.3 \pm 2.25$ \\
\hline
\end{tabular}


individual phase values were computed for every sampled point $i$ in a trial, $\theta_{i}=\tan ^{-1}\left[\left(d x_{i} / d t\right) / x_{i}\right]$, with $x_{i}$ the normalized position and $d x_{i} / d t$ the normalized instantaneous velocity. For each sampled point $i$ in a trial, the continuous relative phase was computed as $\phi_{i}=\theta_{\text {computer }}-\theta_{\text {joystick. }}$. Mean resultant vectors were computed from the observed unit vectors $(x, y)$ of the $\phi_{i}$ values. The mean resultant vectors were used to compute a mean phase angle $\left(\phi_{o b s}\right)$ and a magnitude of circular variance. A value of $\phi_{o b s}=0^{\circ}$ represented in-phase tracking, and a value of $\theta_{o b s}=180^{\circ}$ represented anti-phase tracking. There were three required relative phase values, $\phi_{r e q}=0^{\circ},-90^{\circ}$, and $180^{\circ}$. An absolute phase error $\left(\phi_{A E}=\left|\phi_{\text {req }}-\phi_{\text {obs }}\right|\right)$ was computed and used to evaluate tracking accuracy.

A circular variance was computed to characterize the variability of the joystick trajectory. The circular variance fell within the range of 0 to 1 , with 1 representing perfect uniformity. To submit the circular variance data to inferential tests based on standard normal theory ${ }^{16)}$, the circular variance score was transformed to the range 0 to $\infty$ as follows, $\mathrm{s}_{0}=-2 \log _{\mathrm{n}}\left(1-\mathrm{S}_{0}\right)^{0.5}$. $\mathrm{S}_{0}$ denotes the measure of circular variance for the interval $[0,1]$, with $\mathrm{s}_{0}$ the transformed circular variance (TCV) measure that was submitted for statistical analysis. This TCV measure was treated as an estimate of the pattern stability in tracking performance.

A discrete Fourier transformation was applied to the velocity time series with a Hanning window. The power spectrum of the joystick trajectory's velocity was then computed to analyze the subject's performance in the frequency domain. The power spectrum provided information mainly about the distribution of the power across the frequency component of the signal. To simplify this information, the distribution of the power spectrum was normalized by the total power, so that the total area of the normalized power became 1. The power spectrum also provided the frequency of the primary peak, which was defined as the frequency at the largest power in the spectrum. Three frequency components (lower, main, and higher) were defined to further understand the power distribution of the frequency components. The main frequency component was defined by $\pm 0.0476 \mathrm{~Hz}$ of the frequency of the primary peak. For example, the range of the main component would be $0.285 \mathrm{~Hz}$ to $0.381 \mathrm{~Hz}$, with $0.333 \mathrm{~Hz}$ being the primary peak. The higher frequency component (HFC) was defined by the frequency above the main frequency component. The HFC indicated intermittency in which an increase in corrective movements leads to an increase of the proportion.

Dependent variables were the absolute phase error $\left(\phi_{A E}\right)$, TCV, mean power frequency, and the percentage of power in the HFC (HFC percentage). The four dependent variables were analyzed in a 2 Age (young, old) $\times 3$ Pattern $\left(0^{\circ}, 90^{\circ}, 180^{\circ}\right)$, with the last factor repeated. Duncan's multiple range tests $(\mathrm{p}<0.05)$ were used to analyze all post-hoc main effects. Partial eta squared values $\left(\eta_{\mathrm{p}}^{2}\right)$ were computed and reported with every significant $F$ value.

\section{RESULTS}

Neither main nor interaction was found in the $\phi_{A E}$ data (ps $>0.05$ ). The analysis of the TCV data revealed that elderly subjects $(\mathrm{TCV}=2.23)$ produced significantly more tracking variability compared with young subjects $(\mathrm{TCV}=3.16)\left(F_{(1,10)}\right.$ $\left.=14.27, \mathrm{p}<0.01, \eta_{p}{ }^{2}=0.59\right)$. A significant main effect of Pattern in the TCV values was found $\left(F_{(2,20)}=54.12, \mathrm{p}<0.0001\right.$, $\left.\eta_{p}{ }^{2}=0.84\right)$, and post-hoc tests revealed that the $0^{\circ}(\mathrm{TCV}=3.20)$ and $180^{\circ}(\mathrm{TCV}=3.08)$ tracking patterns were significantly more stable than the $90^{\circ}$ tracking pattern $(\mathrm{TCV}=1.80)$.

Power spectrum analysis showed that the primary peak located at the target frequency and the peak frequency was not significantly affected by age or patterns (ps $>0.5)$. The analysis of the HFC percentage revealed that the elderly subjects $(\mathrm{HFC}=53.4 \%)$ produced more corrective movements than did the young subjects $(\mathrm{HFC}=37.1 \%)\left(F_{(1,10)}=27.47, \mathrm{p}<0.001\right.$, $\left.\eta_{p}{ }^{2}=0.73 .3\right)$. A significant main effect of Pattern in the HFC percentage was found $\left(F_{(2,20)}=4.27, \mathrm{p}<0.05, \eta_{p}{ }^{2}=0.30\right)$, and post-hoc tests revealed that the $0^{\circ}(\mathrm{HFC}=41.9 \%)$ and $180^{\circ}(\mathrm{HFC}=43.5 \%)$ tracking patterns were performed with less corrective movements compared with the $90^{\circ}$ tracking pattern $(\mathrm{HFC}=50.3 \%)$.

\section{DISCUSSION}

In the visuomotor tracking task used in the current study, the elderly and young subjects had to perform $0^{\circ}, 90^{\circ}$, and $180^{\circ}$ tracking patterns. The $0^{\circ}$ and $180^{\circ}$ tracking patterns were stable compared with the $90^{\circ}$ tracking pattern for both age groups. These findings were consistent with the prediction by the HKB model ${ }^{5)}$ and also consistent with many previous visuomotor tracking experiments examining perception-action coordination ${ }^{3,4)}$. The present result also showed that the elderly subjects were less stable than the young subjects for all tracking patterns, consistent with the previous tracking studies ${ }^{14,15)}$.

The differential stability found in the present study can be understood as the difference of perceptual stability created by the visuomotor coordination patterns, in that the $0^{\circ}$ and $180^{\circ}$ patterns were perceived with more coordination than the $90^{\circ}$ tracking pattern ${ }^{17,18)}$. The present visuomotor tracking task was accompanied by a perceptual cost that discriminated coordination between the two dots, and the actor had to perform physical tracking in accordance with the specific coordination relationship while continuously processing the visual feedback. Therefore, any reduction of perceptual ability would inhibit processing of visual information, destabilizing tracking performance. That is, the differential stability among the three coordination patterns used in the current study is thought to have originated from differences between each tracking pattern's perceptual stability ${ }^{17,18)}$. For the same reason, the overall decrease in tracking stability of the elderly suggested an overall loss of perceptual ability with age ${ }^{14}$. 
The elderly showed more corrective movements than did the young subjects in the current study. Since the degree of corrective movements is determined by the utilization of visual feedback information, it is reasonable to assume that the elderly subjects used more visual feedback than did the young subjects. This might be due to the decrease of accuracy and stability of tracking performance resulting from the degradation of perceptual ability with age. In turn, the degraded tracking performance required the elderly to adjust tracking movements frequently through increased utilization of feedback information. Unlike the elderly, the young subjects required less perceptual effort to discriminate coordination patterns due to stable perception, leading in turn to fewer corrective movements.

An interesting finding was that the $90^{\circ}$ tracking pattern was performed with the most corrective movements compared with the other two patterns, suggesting that the degree of intermittency can depend on the degree of perceptual stability. That is, the $90^{\circ}$ tracking pattern was a perceptually unstable coordination pattern, requiring more discrimination effort than the other 2 patterns, resulting in increased corrective movements. In the stable $0^{\circ}$ and $180^{\circ}$ tracking patterns, the coordination patterns were relatively easy to perceive, so fewer corrective movements were required compared with the unstable $90^{\circ}$ pattern. The $90^{\circ}$ pattern is a perceptually unstable pattern, thus corrective movements increased through more visual feedback.

Taken together, the present findings indicate that the intrinsic coordination dynamics predicted by the HKB model did not change with age, whereas utilization of visual feedback information declined overall ${ }^{19)}$. Therefore, the decline in perceptionaction coordination skills is related to the lower utilization of visual feedback information. The number of subjects participating in this study might be too small to make generalized conclusions from the data. Further research is needed on the methods for increasing the utilization of visual feedback information from the perspective of rehabilitation.

\section{ACKNOWLEDGEMENTS}

This study was supported by Basic Science Research Program through the National Research Foundation of Korea (NRF) funded by the Ministry of Education, Science and Technology (2010-0006539) and by the National Research Foundation of Korea (NRF) Grant funded by the Korean Government (MSIP) (No.2015R1A5A7037630).

\section{REFERENCES}

1) Wimmers RH, Beek PJ, Van Wieringen PC: Phase-transitions in rhythmic tracking movements-a case of unilateral coupling. Hum Mov Sci, 1992, 11: 217-226. [CrossRef]

2) Shin HK, Park JW, Ryu YU: Influence of movement speed on accuracy of tracking performance following stroke. $\mathrm{J}$ Phys Ther Sci, 2011, 23: 141-144. [CrossRef]

3) Ryu YU, Buchanan JJ: Learning an environment-actor coordination skill: visuomotor transformation and coherency of perceptual structure. Exp Brain Res, 2009, 196: 279-293. [Medline] [CrossRef]

4) Ryu YU, Buchanan JJ: Accuracy, stability, and corrective behavior in a visuomotor tracking task: a preliminary study. PLoS ONE, 2012, 7: e38537. [Medline] [CrossRef]

5) Haken H, Kelso JA, Bunz H: A theoretical model of phase transitions in human hand movements. Biol Cybern, 1985, 51: 347-356. [Medline] [CrossRef]

6) McNay EC, Willingham DB: Deficit in learning of a motor skill requiring strategy, but not of perceptuomotor recalibration, with aging. Learn Mem, 1998, 4: 411-420. [Medline] [CrossRef]

7) Song CS: Relationship between visuo-perceptual function and manual dexterity in community-dwelling older adults. J Phys Ther Sci, 2015, 27: 1871-1874. [Medline] [CrossRef]

8) Seidler RD: Differential effects of age on sequence learning and sensorimotor adaptation. Brain Res Bull, 2006, 70: 337-346. [Medline] [CrossRef]

9) Seidler-Dobrin RD, Stelmach GE: Persistence in visual feedback control by the elderly. Exp Brain Res, 1998, 119: 467-474. [Medline] [CrossRef]

10) Verwey WB: Diminished motor skill development in elderly: indications for limited motor chunk use. Acta Psychol (Amst), 2010, 134: 206-214. [Medline] [CrossRef]

11) Sosnoff JJ, Newell KM: Are visual feedback delays responsible for aging-related increases in force variability? Exp Aging Res, 2007, 33: 399-415. [Medline] [CrossRef]

12) Tracy BL, Dinenno DV, Jorgensen B, et al.: Aging, visuomotor correction, and force fluctuations in large muscles. Med Sci Sports Exerc, 2007, 39: 469-479. [Medline] [CrossRef]

13) Russell DM, Sternad D: Sinusoidal visuomotor tracking: intermittent servo-control or coupled oscillations? J Mot Be- 
hav, 2001, 33: 329-349. [Medline] [CrossRef]

14) Coats RO, Snapp-Childs W, Wilson AD, et al.: Perceptuo-motor learning rate declines by half from 20 s to $70 / 80$ s. Exp Brain Res, 2013, 225: 75-84. [Medline] [CrossRef]

15) Jagacinski RJ, Liao MJ, Fayyad EA: Generalized slowing in sinusoidal tracking by older adults. Psychol Aging, 1995, 10: 8-19. [Medline] [CrossRef]

16) Burgess-Limerick R, Abernethy B, Neal RJ: Note: a statistical problem in testing invariance of movement using the phase plane model. J Mot Behav, 1991, 23: 301-303. [Medline] [CrossRef]

17) Bingham GP, Schmidt RC, Zaal FT: Visual perception of the relative phasing of human limb movements. Percept Psychophys, 1999, 61: 246-258. [Medline] [CrossRef]

18) Zaal FT, Bingham GP, Schmidt RC: Visual perception of mean relative phase and phase variability. J Exp Psychol Hum Percept Perform, 2000, 26: 1209-1220. [Medline] [CrossRef]

19) Lee NK, Kwon YH, Son SM, et al.: The effects of aging on visuomotor coordination and proprioceptive function in the upper limb. J Phys Ther Sci, 2013, 25: 627-629. [Medline] [CrossRef] 